Proceedings of the Edinburgh Mathematical Society (2005) 48, 219-229 (C)

DOI:10.1017/S0013091504000719 Printed in the United Kingdom

\title{
MULTIPLICATIVELY SPECTRUM-PRESERVING MAPS OF FUNCTION ALGEBRAS. II
}

\author{
N. V. RAO ${ }^{1}$ AND A. K. ROY ${ }^{2}$ \\ ${ }^{1}$ Mathematics Department, University of Toledo, Toledo, \\ OH 43606, USA (rnagise@math.utoledo.edu) \\ ${ }^{2}$ Indian Statistical Institute-Calcutta, Statistics and Mathematics Unit, \\ 203 BT Road, Calcutta 700 108, India (ashoke@isical.ac.in)
}

(Received 27 July 2004)

\begin{abstract}
Let $\mathcal{A}$ be a closed, point-separating sub-algebra of $C_{0}(X)$, where $X$ is a locally compact Hausdorff space. Assume that $X$ is the maximal ideal space of $\mathcal{A}$. If $f \in \mathcal{A}$, the set $f(X) \cup\{0\}$ is denoted by $\sigma(f)$. After characterizing the points of the Choquet boundary as strong boundary points, we use this equivalence to provide a natural extension of the theorem in [10], which, in turn, was inspired by the main result in [6], by proving the 'Main Theorem': if $\Phi: \mathcal{A} \rightarrow \mathcal{A}$ is a surjective map with the property that $\sigma(f g)=\sigma(\Phi(f) \Phi(g))$ for every pair of functions $f, g \in \mathcal{A}$, then there is an onto homeomorphism $\Lambda: X \rightarrow X$ and a signum function $\epsilon(x)$ on $X$ such that
\end{abstract}

$$
\Phi(f)(\Lambda(x))=\epsilon(x) f(x)
$$

for all $x \in X$ and $f \in \mathcal{A}$.

Keywords: automorphism; function algebra; spectrum; boundaries

2000 Mathematics subject classification: Primary 46J10; 46J20

\section{Introduction}

If $X$ is a locally compact Hausdorff space, we let $C_{0}(X)$ denote the classical Banach algebra of continuous complex-valued functions on $X$ vanishing at infinity and equipped with the supnorm $\|f\|_{\infty}:=\sup \{|f(x)|: x \in X\}$. Our main concern here is with subalgebras $\mathcal{A}$ of $C_{0}(X)$, which are closed in the supnorm topology defined above and which are point separating in the sense that, given $x, y \in X$ with $x \neq y$, there is an $f \in \mathcal{A}$ with $f(x) \neq f(y)$. Such objects $\mathcal{A}$ we also designate as function algebras as in [10], the only difference being that the algebras considered here do not contain constants.

If $X_{\infty}=X \cup\{\infty\}$ is the one-point compactification of $X$, let

$$
\mathcal{A}^{\prime}=\{f+\lambda: f \in \mathcal{A}, \lambda \in \mathbb{C}\} .
$$

One verifies easily that $\mathcal{A}^{\prime}$ is a (supnorm) closed, point-separating subalgebra of $C\left(X_{\infty}\right)$ containing constants. In the next section, we make use of facts known about $\mathcal{A}^{\prime}$ (for 
example, that $\mathcal{A}$ is a closed ideal of $\mathcal{A}^{\prime}$ ) to derive characterizations of members of $\partial_{\mathcal{A}}(X)$, the so-called Choquet boundary of $\mathcal{A}$, which we now proceed to define.

If we denote by $\operatorname{Ext}(B)$ the non-empty set of extreme points of the weak*-compact unit ball $B$ of the dual $\mathcal{A}^{*}$ of $\mathcal{A}$, then it is known that

$$
\operatorname{Ext}(B) \subseteq\left\{\alpha e_{x}:|\alpha|=1, x \in X\right\},
$$

where $e_{x}$ is the point evaluation at $x$,

$$
e_{x}(f)=f(x), \quad f \in \mathcal{A}
$$

(see [4, p. 441]). If we let $e$ be the map $x \rightsquigarrow e_{x}$ from $X$ to $B$, we can define

$$
\partial_{\mathcal{A}}(X)=e^{-1}(\operatorname{Ext}(B))
$$

(see $[\mathbf{1}, \mathbf{5}, \mathbf{8}]) . \overline{\partial_{\mathcal{A}}(X)}$ is the usual Shilov boundary of $\mathcal{A}$.

We define peaking functions and generalized peak points for the function algebra $\mathcal{A}$ as in [10]. Let us note in passing that, in [1] , an $x_{0} \in X$ is called a 'strong boundary point' if, given any neighbourhood $U$ of $x_{0}$, there exists $f_{1} \in A$ with $\left\|f_{1}\right\|_{\infty}=1$ and $M_{f_{1}}:=\left\{x:\left|f_{1}(x)\right|=1\right\} \subset U$ (so that $\left|f_{1}\right|<1$ off $U$.) This conforms to our usage of the term 'generalized peak point' in [10]: one simply observes that if $x_{0} \in M_{f_{1}}$ and $f_{1}\left(x_{0}\right)=\mathrm{e}^{\mathrm{i} \theta_{0}}$, and we define $g_{1}=\mathrm{e}^{-\mathrm{i} \theta_{0}} f_{1} \in \mathcal{A}$, then the function $f=g_{1} \mathrm{e}^{g_{1}} / \mathrm{e}$ defines a peaking set $P(f):=\{f=1\} \subset U, x_{0} \in P(f)$, and $|f|<1$ off $P(f)\left(f \in \mathcal{A}\right.$, since $g_{1} \in \mathcal{A}$, $e^{g_{1}} \in \mathcal{A}^{\prime}$, and $\mathcal{A}$ is an ideal in $\left.\mathcal{A}^{\prime}\right)$.

We extend and complete the results in $[\mathbf{1}]$ by proving in $\S 2$ that $x \in \partial_{\mathcal{A}}(X)$ if and only if $x$ is a generalized peak point (or, equivalently, a strong boundary point). The proof will be based on the following analogue, for $\mathcal{A}$, of a theorem of Bishop well known in the context of uniform algebras (see (1.5) in $[\mathbf{1 0}]$ and the references given there).

Theorem 1.1. Let $K$ be a peak set in $X$ of a function $h \in \mathcal{A}$ (or $\left.\mathcal{A}^{\prime}\right)$. Suppose that $g \in \mathcal{A}$ and $g \not \equiv 0$ on $K$. Then there exists an $f \in \mathcal{A}$ with $\left.f\right|_{K}=\left.g\right|_{K}$ and such that $|f(x)|<\|f\|_{\infty}$ for all $x \in X \backslash K$.

The proof is omitted as it is the same, with obvious modifications, as that given in [3]. We will only emphasize here that the function $f$ in the above theorem is defined by $f=g \sum_{n=1}^{\infty} 2^{-n} h^{k_{n}}$, for suitably large positive integers $k_{n}$, and thus $f \in \mathcal{A}$ even if $h \in \mathcal{A}^{\prime}$.

Using all these results and some facts recorded in $\S 2$, we finally prove in $\S 3$ of the paper our main theorem.

Main Theorem. Let $\sigma(f):=f(X) \cup\{0\}$ for $f \in \mathcal{A}$. Assume that $X$ is the maximal ideal space of $\mathcal{A}$. If $\Phi: \mathcal{A} \rightarrow \mathcal{A}$ is a surjective map with the property that

$$
\sigma(f g)=\sigma(\Phi(f) \Phi(g))
$$

for every pair of functions $f, g \in \mathcal{A}$, then there exists a homeomorphism $\Lambda$ of $X$ onto $X$ and a signum function $\epsilon(x)$ on $X$ (i.e. $\epsilon(x)= \pm 1$ for all $x \in X$ ) such that

$$
\Phi(f)(\Lambda(x))=\epsilon(x) f(x) \quad \forall f \in \mathcal{A}, x \in X .
$$


This is the natural extension of the theorem proved in $[\mathbf{1 0}]$, which, in turn, was a generalization of Theorem 5 in $[\boldsymbol{6}]$.

All unexplained notations and terminology will be found in $[\mathbf{1 0}]$.

\section{Characterizing the Choquet boundary and some miscellaneous facts}

We now prove the following theorem (probably known but we could not find a reference).

Theorem 2.1. For the function algebra $\mathcal{A} \subseteq C_{0}(X)$, the following statements are equivalent.

(a) $x \in \partial_{\mathcal{A}}(X)$.

(b) $x$ is a strong boundary point.

Proof. $(\mathrm{a}) \Rightarrow$ (b) Suppose that $x \in \partial_{\mathcal{A}}(X)$. From the definition of $\partial_{\mathcal{A}}(X), e_{x} \in B$ and $\left\|e_{x}\right\|=1$, with the latter norm being the norm on the dual $\mathcal{A}^{*}$.

We first claim that $x \in \partial_{\mathcal{A}^{\prime}}\left(X_{\infty}\right)$. To see this, let $\mu$ be a Borel probability measure representing $x$ on $\mathcal{A}^{\prime}$, i.e.

$$
f(x)+\lambda=\int_{X_{\infty}}(f+\lambda) \mathrm{d} \mu, \quad f \in \mathcal{A}, \quad \lambda \in \mathbb{C} .
$$

By a standard result in convexity theory (see $\left[8\right.$, p. 38]), we have to show that $\mu=\delta_{x}$, the unit point mass at $x$. From $(*)$, it is evident that

$$
f(x)=\int_{X_{\infty}} f \mathrm{~d} \mu \quad \forall f \in \mathcal{A} .
$$

Writing $\mu=\mu \mid X+\mu(\infty) \delta_{\infty}$, this means that

$$
f(x)=\int_{X} f \mathrm{~d} \mu+\mu(\infty) f(\infty)=\int_{X} f \mathrm{~d} \mu, \quad f \in \mathcal{A},
$$

and thus

$$
\begin{aligned}
1 & =\left\|e_{x}\right\| \\
& =\sup \left\{|f(x)|: f \in \mathcal{A},\|f\|_{\infty} \leqslant 1\right\} \\
& =\sup \left\{\left|\int_{X} f \mathrm{~d} \mu\right|: f \in \mathcal{A},\|f\|_{\infty} \leqslant 1\right\} \\
& \leqslant \mu(X) \\
& \leqslant 1
\end{aligned}
$$

showing that $\mu(\infty)=0$. Hence $\mu$ is concentrated on $X$ and, being a representing measure for $x$ on $\mathcal{A}$ and because $x \in \partial_{\mathcal{A}}(X), \mu=\delta_{x}$ by [9, Proposition 3.7]. Since $x \in \partial_{\mathcal{A}^{\prime}}\left(X_{\infty}\right), x$ is a generalized peak point for $\mathcal{A}^{\prime}$, as $\mathcal{A}^{\prime}$ is an algebra containing constants (see [8, p. 37]). If $U$ is a neighbourhood of $x$ in $X$, there exists a function $g \in \mathcal{A}^{\prime}$ 
such that the set $E:=\{y: g(y)=1\} \subset U, x \in E$ and $|g|<1$ off $E$. Let $\lambda=g(\infty)$. Notice that $|\lambda|<1$ and $g-\lambda \in \mathcal{A}$. Let $f=(g-\lambda) /(1-\bar{\lambda} g)$. It is easy to see that $f \in \mathcal{A}$ and $|f|<1$ off $E$ and $f$ is equal to $(1-\lambda) /(1-\bar{\lambda})$ on $E$ with absolute value 1 , and therefore $x$ is a generalized peak point for $\mathcal{A}$. Alternatively, we can construct such functions by a simple application of Theorem 1.1.

(b) $\Rightarrow$ (a) Let $x \in X$ be a strong boundary point and let $U$ be a neighbourhood of $x$. Then there exists an $f \in \mathcal{A}$ such that $\|f\|_{\infty}=1=f(x)$ and $|f|<1$ off $U$. According to [9, Proposition 3.7], we have to prove that if $\mu$ is any complex regular Borel measure on $X_{\infty}$ such that $\|\mu\|_{\infty} \leqslant 1$ and $\mu$ represents $x$ on $\mathcal{A}$, then $\mu=\delta_{x}$, the unit point mass at $x$. Let $|\mu|$ denote the total variation measure of $\mu$. Now, for any positive integer $n$, we have

$$
1=\left|f^{n}(x)\right|=\left|\int_{X_{\infty}} f^{n} \mathrm{~d} \mu\right| \leqslant|\mu|(U)+\int_{X_{\infty} \backslash U}\left|f^{n}\right| \mathrm{d}|\mu| .
$$

By letting $n \rightarrow \infty$, we obtain $|\mu|(U)=1$, and so $|\mu|\left(X_{\infty} \backslash U\right)=0$. Since $U$ is arbitrary, we obtain that $|\mu|=\delta_{x}$. But then $\mu=\gamma \delta_{x}$ with $|\gamma|=1$. As $f(x)=\gamma f(x) \forall f \in \mathcal{A}$ and $\left\|e_{x}\right\|=1$, there exists $f \in \mathcal{A}$ with $f(x) \neq 0$, and hence $\gamma=1$, proving $\mu=\delta_{x}$. This concludes the proof of Theorem 2.1.

\section{1.}

We now record a few observations that will be needed in the proof of the Main Theorem in the next section.

(1) A peaking set meets $\partial_{\mathcal{A}}(X)$.

This follows from the fact that $\partial_{\mathcal{A}}(X)$ is a boundary for $\mathcal{A}$.

(2) Any family of peaking sets with non-empty intersection contains a point of $\partial_{\mathcal{A}}(X)$.

This has the same proof as that of Proposition 1.6. in $[\mathbf{1 0}]$ with the state space there being replaced by the weak*-compact unit ball $B$ of $\mathcal{A}^{*}$, the dual space of $\mathcal{A}$.

(3) Given $x \in X$, there exists a Borel probability measure $\mu$ supported on the Shilov boundary $S=\overline{\partial_{\mathcal{A}}(X)}$ and representing $x$,

$$
f(x)=\int_{S} f \mathrm{~d} \mu, \quad f \in \mathcal{A} .
$$

This fact, which is easy to prove for algebras containing constants, has a somewhat non-trivial proof (see [11, p. 106]).

(4) The function algebra $\mathcal{A}$ is strongly separating, i.e. $\{|f|: f \in \mathcal{A}\}$ separates points of $X$.

This is, of course, well known and easy to prove for algebras with constants, but in the present context follows from [9, Proposition $3.4(\mathrm{vi})]$ and shows that the assumption of strong separation made in the main result of [1, Theorem 5, p. 82] is redundant-point separation by $\mathcal{A}$ suffices. 
(5) If $X$ is the maximal ideal space of $\mathcal{A}$, as stated above in the Main Theorem, then $X_{\infty}$ is the maximal ideal space of $\mathcal{A}^{\prime}$ and if, for $f \in \mathcal{A}, \sigma_{\mathcal{A}}(f)$ (respectively, $\sigma_{\mathcal{A}^{\prime}}(f)$ ) denotes the spectrum of $f$ as an element of $\mathcal{A}$ (respectively, $\mathcal{A}^{\prime}$ ), then

$$
\sigma_{\mathcal{A}}(f)=\sigma_{\mathcal{A}^{\prime}}(f)=\text { range of } f \text { on } X_{\infty}=f(X) \cup\{0\} .
$$

This follows from standard Banach algebra theory expounded, for instance, in $[\mathbf{2}$, Lemma B.4.2, p. 307]. This explains the reason for the notation $\sigma(f)$, and the assumption concerning $X$, in the abstract.

\section{Proof of the Main Theorem}

In the present section, we will prove the main theorem stated in the abstract. We let $f, g$, $h, k$ denote functions from $\mathcal{A}$ and let $c$ stand for a generic constant. We shall sometimes write $\|f\|$ instead of $\|f\|_{\infty}$. As in [10], the proof is most conveniently presented through a series of remarks. We should point out that the proofs of several of these remarks are almost identical to the corresponding ones in [10], though our algebra $\mathcal{A}$ does not contain the constants and are therefore omitted. Nevertheless, for ease of reading, we include full statements of these remarks and refer to the appropriate places in [10] where their proofs may be found. And, of course, we point out the differences, from [10], in the proofs of the other remarks caused by the absence of scalars.

Remark 3.1. We have

$$
\sigma\left(f^{2}\right)=\sigma\left(\Phi(f)^{2}\right) \quad \forall f \in \mathcal{A},
$$

from which it immediately follows that $\sigma(|f|)=\sigma(|\Phi(f)|)$ and

$$
\|f\|_{\infty}=\|\Phi(f)\|_{\infty}
$$

Note that, unlike [10], we cannot make the reduction $\sigma(f)=\sigma(\Phi(f)) \forall f \in \mathcal{A}$.

Remark 3.2. If $f, g \in \mathcal{A}$, then $|f| \leqslant|g|$ on $\partial_{\mathcal{A}}(X)$ if and only if

$$
\text { for every } c \geqslant 0 \text { and every } h,|g h| \leqslant c \text { implies }|f h| \leqslant c \text {. }
$$

The proof is omitted since it is very similar to Remark 2 of [10], despite the absence of constants in $\mathcal{A}$.

From Remark 3.2, we can deduce the following:

$$
\text { if } \sigma(f h)=\sigma(g h) \text { for every } h \text {, then }|f|=|g| \text { on } \partial_{\mathcal{A}}(X) \text {. }
$$

As $\sigma(f h)=\sigma(g h) \forall h \in \mathcal{A}$, we see that, for any constant $c \geqslant 0$ and any $h \in \mathcal{A},|g h| \leqslant c$ implies $|f h| \leqslant c$. So Remark 3.2 gives $|f| \leqslant|g|$ on $\partial_{\mathcal{A}}(X)$. Since the hypothesis is symmetric in $f, g$, we also obtain $|g| \leqslant|f|$ on $\partial_{\mathcal{A}}(X)$. Combining, we have (3.4).

As a consequence, we have the following. 
Remark 3.3. We have

$$
|f| \leqslant|g| \quad \text { on } \partial_{\mathcal{A}}(X) \quad \Leftrightarrow \quad|\Phi(f)| \leqslant|\Phi(g)| \quad \text { on } \partial_{\mathcal{A}}(X) \quad \forall f, g \in \mathcal{A} .
$$

We omit the proof, since it is the same as that of Remark 3 of [10].

Remark 3.4. For any fixed $x \in \partial_{\mathcal{A}}(X)$,

$$
E:=\bigcap_{f \in \mathcal{F}_{x}} M(f)=\{x\}
$$

where $M(f):=\{t \in X:|f(t)|=\|f\|=1\}, \mathcal{F}_{x}$ denotes the family of all functions $f \in A$ such that $x \in M(f)$ and we refer to the latter set as the $M$-set for $f$.

Proof. Assume that $E$ contains a point $y$ other than $x$. From Theorem 2.1, it follows that every point of $\partial_{\mathcal{A}}(X)$ is a generalized peak point for $\mathcal{A}$, which means that, given any neighbourhood $V$ of $x$, there exists a peaking function $h$ in $\mathcal{A}$ such that $h(x)=1=\|h\|$ and $|h|<1$ outside $V$, which means that $P(h) \subset V$. So, if we choose a neighbourhood $V$ of $x$ that does not contain $y$, since $P(h) \subset V, y \notin E$, a contradiction.

We now have the following important result.

Remark 3.5. If $x \in \partial_{\mathcal{A}}(X)$, then

$$
\bigcap_{f \in \mathcal{F}_{x}} M(\Phi(f)) \text { contains one and only one generalized peak point. }
$$

Proof. The proof is similar to that of Remark 5 of [10]. However, since the result is so crucial for what follows, we reproduce the proof with due care.

We notice that $M(\Phi(f))$ is compact. Secondly, if $f_{1}, f_{2}, \ldots, f_{n}$ belong to $\mathcal{F}_{x}$, then $g=f_{1} f_{2} \cdots f_{n}$ belongs to $\mathcal{F}_{x}$. Since $|g| \leqslant\left|f_{i}\right|$, we obtain, in view of $(3.5)$,

$$
|\Phi(g)| \leqslant\left|\Phi\left(f_{i}\right)\right| \quad \text { on } \partial_{\mathcal{A}}(X) \text { for each } 1 \leqslant i \leqslant n .
$$

Since $\|g\|=1$, we have that $\|\Phi(g)\|=1$ by Remark 3.1 and $|\Phi(g)(\xi)|=1$ for some $\xi$ in $\partial_{\mathcal{A}}(X)$. Then $\left|\Phi\left(f_{i}\right)(\xi)\right|=1$ because $\left\|f_{i}\right\|=\left\|\Phi\left(f_{i}\right)\right\|=1$ for $1 \leqslant i \leqslant n$, so

$$
\bigcap_{1 \leqslant i \leqslant n} M\left(\Phi\left(f_{i}\right)\right) \neq \emptyset
$$

This proves that the family of sets $\left\{M(\Phi(f)): f \in \mathcal{F}_{x}\right\}$ has finite intersection property and since each of them is compact, it must be that

$$
E^{\prime}=\bigcap_{f \in \mathcal{F}_{x}} M(\Phi(f)) \neq \emptyset .
$$

If $p \in E^{\prime}$, we can, by the device explained in $\S 1$, replace each $M(\Phi(f))$ by the associated peaking set contained in it and containing $p$. Now we can use observation (2) of $\S 2.1$ to see that $E^{\prime}$ must intersect $\partial_{\mathcal{A}}(X)$. 
Thirdly, if $y \in E^{\prime} \cap \partial_{\mathcal{A}}(X)$, let $k \in \mathcal{F}_{y}$. By the surjectivity of $\Phi, k=\Phi(h)$ for some function $h \in \mathcal{A}$ (recall that $\sigma\left(k^{2}\right)=\sigma\left(h^{2}\right)$ ). We claim that $|h(x)|=1$. To show this, choose any neighbourhood $V$ of $x$ and a function $g$ such that $|g(x)|=1$ and $|g|<1$ outside $V$. So $g \in \mathcal{F}_{x}$, and hence $|\Phi(g)(y)|=1$. Consider $\Phi(g) \Phi(h)=\lambda \in \mathcal{A}$. Since $\Phi(g)$, $\Phi(h)$ attain their maximum modulus 1 at $y$, we see that $|\lambda(y)|=1=\|\lambda\|$. Again, $\Phi$ being surjective, there exists a function $\mu \in \mathcal{A}$ such that $\Phi(\mu)=\lambda$. Since $|\lambda| \leqslant|\Phi(g)| \wedge|\Phi(h)|$ on $\partial_{\mathcal{A}}(X)$, by (3.5), it follows that $|\mu| \leqslant|g| \wedge|h|$ on $\partial_{\mathcal{A}}(X)$. But there exists a $\xi$ in $\partial_{\mathcal{A}}(X)$ such that $|\mu(\xi)|=1$, and so $|g(\xi)|=|h(\xi)|=1$, which implies that $\xi \in V$. Since $V$ is an arbitrary neighbourhood of $x$ and $h$ is continuous, we get

$$
|h(x)|=1 .
$$

Lastly, if there is a generalized peak point $z$ other than $y$ in $E^{\prime}$, we can choose $k$ in such a way that $|k(y)|=1,|k(z)|<1$. $\Phi$ being surjective, we obtain $h^{\prime}$ such that $\Phi\left(h^{\prime}\right)=k$. So, by the previous paragraph, we see that $h^{\prime}$ belongs to $\mathcal{F}_{x}$, and so $\left|\Phi\left(h^{\prime}\right)\right|=1$ on $E^{\prime}$ and, consequently, $|k(z)|=1$, which is a contradiction. This proves Remark 3.5.

Let the unique point $y$ obtained in Remark 3.5 be denoted by $\tau(x)$, since it depends on $x$ and nothing else. We sum up what we established above as follows.

Remark 3.6. If $x \in \partial_{\mathcal{A}}(X)$ and $f \in \mathcal{F}_{x}$, then $\tau(x) \in \partial_{\mathcal{A}}(X)$ and $\Phi(f)$ belongs to $\mathcal{F}_{\tau(x)}$. Conversely, if $k \in \mathcal{F}_{\tau(x)}$ and $\Phi(h)=k$, then $h \in \mathcal{F}_{x}$.

We now have the following.

Remark 3.7. $\Phi$ is injective and homogeneous, i.e. $\Phi(c f)=c \Phi(f)$ for any $f \in \mathcal{A}$ and $c \in \mathbb{C}$.

We omit the proof, since it is similar to that of Remark 7 of [10].

Remark 3.8. We have

$$
|f(x)|=|\Phi(f)(\tau(x))| \quad \forall f \in \mathcal{A} \quad \forall x \in \partial_{\mathcal{A}}(X) .
$$

We omit the proof as it is similar to that of Remark 8 of $[\mathbf{1 0}]$.

Remark 3.9. $\tau$ is a homeomorphism of $\partial_{\mathcal{A}}(X)$ onto itself.

Proof. We observe first that $\tau$ is injective. If $\tau(x)=\tau(y)$, then $|\Phi(f)(\tau(x))|=$ $|\Phi(f)(\tau(y))|$, and this implies that $|f(x)|=|f(y)|$ for all $f \in \mathcal{A}$ by Remark 3.8. By observation (4) of $\S 2.1$, we see that $x=y$. Next we show that $\tau$ is continuous. Choose any $x \in \partial_{\mathcal{A}}(X)$, a neighbourhood $V$ of $\tau(x)$ and a peaking function $h$ such that

$$
h(\tau(x))=1,|h(y)| \leqslant \frac{1}{2} \quad \forall y, \quad X \backslash V .
$$

With $\Phi$ being surjective, there exists a $g$ such that $\Phi(g)=h$. Since $|g| \equiv|\Phi(g(\tau))|$ by Remark 3.8, if we let $W=\left\{\xi:|g(\xi)|>\frac{1}{2}\right\}$, then $\tau(W) \subset V$ because, if $\xi \in W$, then

$$
|g(\xi)|=|\Phi(g)(\tau(\xi))|=|h(\tau(\xi))|>\frac{1}{2} .
$$


Since $|g(x)|=|\Phi(g)(\tau(x))|=|h(\tau(x))|=1, W$ is a neighbourhood of $x$. Thus we have proved that $\tau$ is injective and continuous.

Now, since $\Phi$ is a bijection, we see that $\Phi^{-1}$ has the same properties as $\Phi$. Thus there would exist an injective continuous map $\psi: \partial_{\mathcal{A}}(X) \rightarrow \partial_{\mathcal{A}}(X)$ such that

$$
|g(x)| \equiv|h(\psi(x))| \quad \forall x \in \partial_{\mathcal{A}}(X) \quad \forall g \in \mathcal{A} .
$$

Let $g=\Phi(h)$. Then $|\Phi(h)(x)|=|h(\psi(x))|$. Let $x=\tau(y)$. Then $|h(y)|=|\Phi(h)(\tau(y))|=$ $|h(\psi(\tau(y)))|$ by Remark 3.8. Since functions of type $|h|$ separate points of $\partial_{\mathcal{A}}(X)$, we get $\psi(\tau(y)) \equiv y$ and, by a similar argument, we also obtain $\tau(\psi(y)) \equiv y$. Thus we have proved that $\tau$ is a self-homeomorphism of $\partial_{\mathcal{A}}(X)$.

The next two remarks provide the most significant point of departure from the proof given in $[\mathbf{1 0}]$.

Remark 3.10. We define a function $\epsilon(x)$ on $\partial_{\mathcal{A}}(X)$ as follows. For a given $x \in$ $\partial_{\mathcal{A}}(X)$, choose any peaking function $h$ such that $h(x)=1$. Notice that $h^{2}$ is also a peaking function and, since $\sigma\left(h^{2}\right)=\sigma\left(\Phi(h)^{2}\right), \Phi(h)^{2}$ is a peaking function and, since $|\Phi(h)(\tau(x))|=h(x)=1$, we see that $\Phi(h)(\tau(x))= \pm$. We define

$$
\epsilon(x)=\Phi(h)(\tau(x)) .
$$

This is independent of the $h$ that is used for its definition: if $h_{1}, h_{2}$ are two peaking functions with $h_{1}(x)=h_{2}(x)=1$, then $\left|\Phi\left(h_{1}\right)(\tau(x)) \Phi\left(h_{2}\right)(\tau(x))\right|=1$ and, since $\sigma\left(h_{1} h_{2}\right)=\sigma\left(\Phi\left(h_{1}\right) \Phi\left(h_{2}\right)\right)$, then $\Phi\left(h_{1}\right) \Phi\left(h_{2}\right)$ is a peaking function because $h_{1} h_{2}$ is and therefore $\Phi\left(h_{1}\right)(\tau(x))=\Phi\left(h_{2}\right)(\tau(x))$.

Remark 3.11. We have

$$
f(x)=\epsilon(x) \Phi(f)(\tau(x)) \text { for all } x \text { in } \partial_{\mathcal{A}}(X) \text { and for all } f \text { in } \mathcal{A} .
$$

Choose any point $x$ in $\partial_{\mathcal{A}}(X)$. Let $V$ be any open neighbourhood of $x$. Since $x$ is in $\partial_{\mathcal{A}}(X)$, there exists a peaking function $h$ such that $h(x)=1$ and the peaking set $P(h)=E$ is contained in $V$. Now, by Bishop's Theorem 1.1, we can modify $h$ so that it has the same properties as before, but, in addition,

$$
|f(z) h(z)|<\max _{E}|f| \quad \text { for all } z \text { outside } E .
$$

Thus there exists a $\xi$ in $E$ such that $|f(\xi)|=\max _{E}|f|=\|f h\|_{\infty}$. Since $\sigma(f h)=$ $\sigma(\Phi(f) \Phi(h))$, we have $\|f h\|=\|\Phi(f) \Phi(h)\|$, and so there exists a point $z$ such that $f(\xi) h(\xi)=\Phi(f)(z) \Phi(h)(z)$. We may assume that $z \in \partial_{\mathcal{A}}(X)$ because the set of points where $\Phi(f) \Phi(h)$ assumes the value $f(\xi) h(\xi)$ is a peaking set and we note that every peaking set meets $\partial_{\mathcal{A}}(X)$.

Since $\tau$ is surjective, $z=\tau(\eta)$ for some $\eta$ in $\partial_{\mathcal{A}}(X)$. Now, by (3.8), we notice that

$$
|\Phi(f)(\tau(\eta)) \Phi(h)(\tau(\eta))|=|f(\eta) h(\eta)| .
$$


Now $\eta$ must be in $E$ because otherwise $|f(\eta) h(\eta)|<|f(\xi)|$ by (3.10). Thus we have found $\xi, \eta$ in $E$ such that $f(\xi)=\Phi(f)(\tau(\eta)) \Phi(h)(\tau(\eta))$. Since $\xi, \eta$ lie in $V$ and $V$ is an arbitrary open neighbourhood of $x$, we get, by continuity of $\tau, f$ and $\Phi(f)$, that $f(x)=\Phi(f)(\tau(x)) \Phi(h)(\tau(x))$, and so, by Remark 3.10, $f(x)=\epsilon(x) \Phi(f)(\tau(x))$. This completes the proof of (3.9).

Remark 3.12. $\Phi$ is a linear isometry of $\mathcal{A}$ onto itself and, furthermore, $\Phi^{2}: \mathcal{A} \rightarrow \mathcal{A}$ is multiplicative.

Proof. We have already seen that $\Phi$ is a bijection and homogeneous. Let $f, g \in \mathcal{A}$. By (3.9), for any $x$ in $\partial_{\mathcal{A}}(X)$,

$$
f(x)=\epsilon(x) \Phi(f)(\tau(x)), \quad g(x)=\epsilon(x) \Phi(g)(\tau(x))
$$

and

$$
f(x) g(x)=\epsilon(x) \Phi(f g)(\tau(x)), \quad f(x)+g(x)=\epsilon(x) \Phi(f+g)(\tau(x)) .
$$

Thus

$\Phi(f g)^{2}(\tau(x))=\Phi(f)^{2}(\tau(x)) \Phi(g)^{2}(\tau(x)), \quad \Phi(f+g)(\tau(x))=\Phi(f)(\tau(x))+\Phi(g)(\tau(x))$.

Since $\tau$ is surjective, we get

$$
\Phi(f)^{2}(x) \Phi(g)^{2}(x)=\Phi(f g)^{2}(x), \quad \Phi(f+g)(x)=\Phi(f)(x)+\Phi(g)(x)
$$

on all of $\partial_{\mathcal{A}}(X)$ and then, by the maximum principle, on all of $X$. This completes the proof of Remark 3.12 .

Finally, we have the following.

Remark 3.13. There exists a self-homeomorphism $\Lambda$ of $X$ onto itself and a function $\gamma(x)$ on $X$ such that $\gamma(x) \equiv \pm 1$ and

$$
\Phi(f)(\Lambda(x))=\gamma(x) f(x) \quad \text { on all of } X .
$$

Corollary 3.14. $\Lambda(x)=\tau(x), \gamma(x)=\epsilon(x)$ for all $x$ in $\partial_{\mathcal{A}}(X)$. This is immediate from (3.9).

Proof of Remark 3.13. We claim that if $M$ is a regular maximal ideal of $\mathcal{A}$, then $N:=\Phi^{-1}(M)$ is also a regular maximal ideal in $\mathcal{A}$. Let $f \in \mathcal{A}, g \in N$. This means that $\Phi(f) \in \mathcal{A}, \Phi(g) \in M$, and so $\Phi^{2}(f) \Phi^{2}(g) \in M$. But $\Phi^{2}(f g)=\Phi^{2}(f) \Phi^{2}(g) \in M$ and, since $M$ is a maximal ideal and hence a prime ideal, $\Phi(f g) \in M$, and so $f g \in N$. Thus we see that $N$ is an ideal, closed of codimension 1 in $\mathcal{A}$, and hence a regular maximal ideal.

Notice that there exists a natural one-to-one correspondence between regular maximal ideals of $\mathcal{A}$ and multiplicative linear functionals on $\mathcal{A}$ of norm less than or equal to 1 , i.e. the points of $X$ by our assumption concerning the maximal ideal space of $\mathcal{A}$. If 
the maximal ideal $M$ is represented by $x \in X$, then the map $e_{x}: \mathcal{A} \rightarrow \mathbb{C}$ defined by $e_{x}(f)=f(x) \forall f \in \mathcal{A}$ satisfies

$$
\operatorname{ker}\left(e_{x}\right)=M
$$

Since $N$ is a maximal ideal and is based on $M$, we denote its representative in $X$ by $\theta(x)$. We now observe that the maps $\Phi^{*}\left(e_{x}\right)=e_{x} \circ \Phi, e_{\theta(x)}: \mathcal{A} \rightarrow \mathbb{C}$ have the same kernel, namely $N$, and hence they differ from each other by a multiplicative constant $\gamma(x)$. So we obtain

$$
e_{x} \circ \Phi=\gamma(x) e_{\theta(x)},
$$

i.e.

$$
f(\theta(x)) \gamma(x)=\Phi(f)(x) \quad \forall x \in X, f \in \mathcal{A} .
$$

Since $\Phi^{2}$ is multiplicative, we have

$$
\Phi^{2}\left(f^{2}\right)=\Phi^{2}(f) \Phi^{2}(f), \quad\left(f^{2}(\theta) \gamma\right)^{2}=(f(\theta) \gamma)^{4},
$$

and consequently, $\gamma^{2}=\gamma^{4} \cdot \gamma= \pm 1$, since $\gamma$ is never zero. Hence we have

$$
|\Phi(f)(x)|=|f(\theta(x))| \quad \forall x \in X, f \in \mathcal{A} .
$$

We claim that $\theta$ is continuous on $X$. Choose any $x$ in $X$ and a net $\left\{x_{\alpha}\right\}$ converging to $x$. Let $\xi$ be any limit point of the net $\left\{\theta\left(x_{\alpha}\right)\right\}$ in $X^{\prime}:=X \cup\{\infty\}$. Since $X^{\prime}$ is compact, such limit points do exist. From (3.12), we conclude that $|\Phi(f)(x)|=|f(\xi)| \forall f \in \mathcal{A}$. Since the family $\{|f|, f \in \mathcal{A}\}$ separates points of $X^{\prime}$, we see that $\xi$ is the only possible limit point and $\xi=\infty$ is not possible since we can choose $f$ so that $\Phi(f)(x) \neq 0$ but $f(\infty)=0$. Therefore, $\theta$ is continuous. Since $\Psi=\Phi^{-1}$ has the same properties as $\Phi$, there exists a continuous function $\nu(x)$ from $X$ to $X$ and a function $\delta(x)= \pm 1$ on $X$ such that

$$
\Psi(f)(x)=\delta(x) f(\nu(x)) \quad \forall x \in X, f \in \mathcal{A} .
$$

Substituting $\Phi(f)$ in place $f$ in the above, we obtain

$$
f(x)=\delta(x) \Phi(f)(\nu(x))=\delta(x) \gamma(\nu(x)) f(\theta(\nu(x))) \quad \forall x \in X, f \in \mathcal{A},
$$

and so $|f(x)| \equiv|f(\theta(\nu(x)))|$. This gives us that $\theta(\nu(x)) \equiv x$ and $\gamma(\nu(x)) \equiv \delta(x)$. Similarly, substituting $\Psi(f)$ in place of $f$ in (3.11), we obtain $\nu(\theta(x)) \equiv x$, proving that $\theta$ is a homeomorphism of $X$ onto itself. Now, if we let $\theta^{-1}=\Lambda$, we get, from (3.11),

$$
\Phi(f)(\Lambda(x))=\gamma(\Lambda(x)) f(x) \quad \forall x \in X, f \in \mathcal{A} .
$$

This completes the proof of the Main Theorem announced in the abstract.

\section{Conclusion}

One could ask whether it is necessary to assume in our Main Theorem that $X$ is the maximal ideal space of $\mathcal{A}$. Indeed, it is necessary. Take, for example, $\mathcal{A}$ to be the algebra of functions of two complex variables $z_{1}, z_{2}$ that are continuous on the closed ball 
$B:=\left\{\left(z_{1}, z_{2}\right):\left|z_{1}\right|^{2}+\left|z_{2}\right|^{2} \leqslant 1\right\}$ and holomorphic in its interior. In this case, the maximal ideal space is the entire closed ball. Let $X$ denote the union of the unit sphere and the point $P=\left(0, \frac{1}{2}\right)$ and $T:=\left(z_{1}, z_{2}\right) \rightsquigarrow\left(z_{1}, \mathrm{i} z_{2}\right)$. Let $\Phi(f)=f \circ T$. This verifies all the hypotheses of the Main Theorem because, for any function $f \in \mathcal{A}, f(B)=f(X)$, otherwise there would exist a value $\alpha$ of $f$ that is assumed in the interior of $B$ but not on $X$, and, in such a case, the solution set of $f(z)=\alpha$ would be a compact complex analytic set and so must be finite (see [7, Corollary 1, p. 55]), a contradiction. But there does not exist any self-homeomorphism $\Lambda$ of $X$ onto itself such that $\Phi(f)=f \circ \Lambda$ because, if it did, $\Lambda\left(0, \frac{1}{2}\right)=T\left(0, \frac{1}{2}\right)$. That is a contradiction because $T\left(0, \frac{1}{2}\right)=\left(0, \frac{1}{2} \mathrm{i}\right)$ does not belong to $X$.

Acknowledgements. A.K.R. thanks the Department of Mathematics of the University of Toledo for its hospitality during the period this paper was written.

\section{References}

1. J. Araujo AND J. Font, On Šilov boundaries for subspaces of continuous functions, Topol. Applic. 77 (1997), 79-85.

2. V. A. Belfi AND R. S. DoRAn, Characterizations of $C *$ algebras: the Gelfand-Naimark theorems (Dekker, New York, 1986).

3. A. Browder, Introduction to function algebras (Benjamin, New York, 1969).

4. N. Dunford and J. Schwartz, Linear operators, Part I (Wiley, 1958).

5. R. Fuhr AND R. Phelps, Uniqueness of complex representing measures on the Choquet boundary, J. Funct. Analysis 14 (1973), 1-27.

6. L. Molnár, Some Characterizations of the automorphisms of $B(H)$ and $C(X)$, Proc. Am. Math. Soc. 130 (2002), 1-11.

7. R. NARAsimhan, Introduction to the theory of analytic spaces, Lecture Notes in Mathematics, vol. 25 (Springer, 1966).

8. R. Phelps, Lectures on Choquet's theorem (Van Nostrand, New York, 1966).

9. R. Phelps, The Choquet representation in the complex case, Bull. Am. Math. Soc. 83 (1977), 299-312.

10. N. V. RAO AND A. K. ROY, Multiplicatively spectrum-preserving maps of function algebras, Proc. Am. Math. Soc., in press.

11. E. L. Stout, The theory of uniform algebras (Bogden \& Quigley, New York, 1971). 